Case Report

\title{
An Exceptional Responder to Nivolumab in Metastatic Non-Small-Cell Lung Cancer: A Case Report and Literature Review of Long-Term Survivors
}

\author{
Babak Baseri $\mathbb{D}^{1},{ }^{1}$ Bachar Samra, ${ }^{1}$ Eric Tam $\mathbb{D}^{1},{ }^{1}$ Edwin Chiu $\mathbb{D}^{1},{ }^{1}$ and Andrea Leaf ${ }^{1,2}$ \\ ${ }^{1}$ Department of Hematology/Oncology, State University of New York Downstate Medical Center, Brooklyn, New York, USA \\ ${ }^{2}$ Department of Hematology/Oncology, Veterans Affairs New York Harbor Healthcare System, Brooklyn Campus, New York, USA
}

Correspondence should be addressed to Babak Baseri; bbaseri.82@gmail.com

Received 9 September 2019; Accepted 4 November 2019; Published 5 December 2019

Academic Editor: Constantine Gennatas

Copyright (C) 2019 Babak Baseri et al. This is an open access article distributed under the Creative Commons Attribution License, which permits unrestricted use, distribution, and reproduction in any medium, provided the original work is properly cited.

\begin{abstract}
Background. Exceptional responders to immune checkpoint inhibitors in metastatic non-small-cell lung cancer (NSCLC) are rare. Furthermore, the optimal duration of immunotherapy in patients who achieve complete remission and the benefit of rechallenge after recurrence remain unknown. Studying the clinical course of exceptional responders can help identify potential predictors of response to immunotherapy and further fine-tune our management algorithms in the absence of standard of care in challenging scenarios. Case Presentation. We highlight the case of a 73-year-old Vietnam War Veteran with active tobacco dependence who achieved complete response with nivolumab for metastatic NSCLC after four prior lines of chemotherapy. Nivolumab was discontinued after 10 cycles due to immune-mediated hepatitis that resolved with steroids. He remained in complete remission for 14 months while off therapy. Then, his tumor recurred twice locally in the mediastinum and he again achieved complete and durable responses after each recurrence with radiotherapy. Due to recurrence in both lungs one year later, he was rechallenged with nivolumab and achieved partial response after two months of therapy. He continues to do well five and a half years since his initial diagnosis of de novo metastatic NSCLC. Conclusion. Optimal management of exceptional responders to immune checkpoint inhibitors in metastatic NSCLC is largely unknown. Our case report adds to the limited data supporting the use of localized therapy for oligometastatic recurrences and rechallenge with immunotherapy for widespread disease in achieving disease control and long-term survival.
\end{abstract}

\section{Introduction}

The use of immune checkpoint inhibitors (ICI) in many malignancies including non-small-cell lung cancer (NSCLC) has revolutionized the field of oncology and has magnified the critical role of the immune system in fighting cancer $[1,2]$. However, only a select group of patients derive clinically meaningful benefit from immunotherapy, ranging from improved quality of life to durable clinical responses, including rare complete remissions that may last many months even beyond immunotherapy discontinuation $[3,4]$. The superior efficacy of immunotherapy in such exceptional responders has sparked an intense research interest in cancer immunobiology $[1,5]$. Here, we describe the clinical course of a patient with heavily pretreated NSCLC who had an exceptional response to a short treatment course with nivolumab.

\section{Case Presentation}

A 73-year-old Vietnam War Veteran with active tobacco dependence ( $1.5-2$ packs per day $>50$ years), prostate cancer in remission (status post definitive radiation in 2008), and alcoholic fatty liver disease was diagnosed in November 2013 with metastatic poorly differentiated lung adenocarcinoma of the left upper lobe (LUL) with biopsy-proven pleural and pericardial metastases after he presented with pneumonia and lung nodules. Molecular studies were negative for EGFR mutation and ALK rearrangement, and nondiagnostic for ROS-1. 
He was started on chemotherapy in January 2014 and received five cycles of carboplatin, pemetrexed, and bevacizumab, followed by three cycles of maintenance pemetrexed and bevacizumab (see Figure 1 for therapy sequence). Due to progression of disease (PD) with new liver lesions, he was switched to second-line docetaxel and he completed six cycles. Although interim positron emission tomography/computed tomography (PET/CT) showed stable disease, the patient developed a paraneoplastic syndrome of inappropriate antidiuretic hormone secretion (SIADH) during the sixth cycle, concerning for PD. Therapy was subsequently switched to erlotinib as third-line therapy. In the interim, the patient reported left shoulder pain that was attributed to a left apical lung tumor involving the pleura and was treated palliatively with RT ( $3000 \mathrm{cGy})$. Notably, hyponatremia resolved within one week of initiating RT, suggesting an abscopal effect given the high burden of disease outside of the radiation field. After three months of receiving erlotinib, PET/CT showed PD but the patient continued to have a good performance status. He was started on fourth-line therapy with vinorelbine and received a total of four cycles until he had recrudescence of SIADH. Imaging showed enlarging hepatic metastasis and left apical and hilar lung lesions, but no evidence of intracranial lesions. Thus, the decision was made to switch therapy to nivolumab $(240 \mathrm{mg}$ IV every two weeks) as fifth line. The patient received 10 cycles from August 2015 to January 2016 and his SIADH resolved after 2 months. Following four months of therapy, nivolumab was held due to grade II transaminitis, for which he was started on prednisone $100 \mathrm{mg}$ daily and had a prolonged steroid taper (for six months). PET/CT following discontinuation of therapy showed no evidence of disease (NED). Nivolumab was not restarted as he was in complete remission, and it was deemed that the risks of nivolumab rechallenge outweighed its benefits. Repeat PET/CT scans (Figure 2) continued to show sustained complete remission, which lasted 14 months after discontinuation of nivolumab, until March 2017 when his tumor recurred in a $1.1 \mathrm{~cm}$ subcarinal node (biopsy proven, PDL-1 positive $80 \%$; $22 \mathrm{C} 3$ pharmDX kit). Molecular studies showed RET rearrangement (10q11) in $84 \%$ of the cells (Leica BioSystems) and were negative for MET amplification and BRAF and HER2 mutations. Given local recurrence with very low disease burden, decision was made to treat the subcarinal node with RT ( $3000 \mathrm{cGy}, 10$ daily fractions of $300 \mathrm{cGy}$ ), with a resultant complete response. Nine months later, PET/CT showed a new hypermetabolic focus (SUV 8.7) in the right paratracheal lymph node (LN, $1.2 \mathrm{~cm}$ from $0.6 \mathrm{~cm}$ previously) and a new hypermetabolic focus (SUV 3.7) in the right middle paratracheal LN $(0.7 \mathrm{~cm})$. Given limited disease, RT was administered to the paratracheal LNs (3000 cGy), and complete response was again achieved until 12 months later, when his disease recurred in the lungs with new and growing bilateral subcentimeter nodules. He was rechallenged with nivolumab and has achieved a partial response after two months of therapy. $\mathrm{He}$ has no evidence of immune-mediated adverse events after 10 cycles. Of note, his SIADH has not recurred since the initial nivolumab therapy, and he continues to be asymptomatic with excellent performance status.

\section{Discussion}

Our case report highlights the oncologic management of a heavily pretreated patient with NSCLC who continues to be alive and well four years since his initial nivolumab treatment. The major benefit in the field of immunooncology in solid tumors, including lung cancer, has been the achievement of durable responses in a subset of patients receiving checkpoint inhibitors, with subsequent translation to longer survivals (Table 1). Most recently, the 5-year follow-up of nivolumab for pretreated advanced solid tumors (up to 5 prior lines of therapy) showed a 5-year overall survival (OS) rate of $16 \%$ across both squamous and nonsquamous NSCLC [6], compared with a historical $4 \%$ rate with traditional cytotoxic therapy [7]. Similarly, the 5-year OS rate for pembrolizumab was recently reported as $15.6 \%$ (previously treated) and $23.2 \%$ (frontline) in the Keynote 001 trial [8]. Furthermore, complete responses (CR) with ICIs are uncommon and have been reported in 1-6\% of NSCLC (Table 1). A large meta-analysis of nine randomized clinical trials evaluating 4803 NSCLC patients treated with ICI reported the incidence of $\mathrm{CR}$ as $1.5 \%$ compared to $0.7 \%$ in chemotherapy groups [3], and found the use of ICIs as first line (relative risk (RR) 2.39, $P=0.032$ ) or second line (RR 4.99, $P=0.038$ ), and the use of nivolumab (RR 4.83, $P=0.042$ ) and atezolizumab (RR 3.26, $P=0.01$ ), but not pembrolizumab and ipilimumab, to be significantly associated with higher $\mathrm{CR}$ rates. However, no correlation was found between OS and CR $(r=0.19, P=0.75)$. Limited data suggest that CRs may be more common in PDL-1-positive tumors and may be associated with the development of irAEs (Table 1) [9].

Predicting which patients will respond to checkpoint inhibitors is an evolving area of research. The current predictive markers of response to immunotherapy in NSCLC remain imperfect and can be divided into two main groups. The first group includes host and tumor/microenvironment factors that already exist prior to the initiation of immunotherapy. These factors include male gender [10], PDL-1 expression $[11,12]$, smoking status $[13,14]$, tumor mutation burden [15], microsatellite instability [16], presence or absence of CD8+ tumor-infiltrating lymphocytes (TILs) [11, 17], KRAS mutation or EGFR wild type [18], previous radiotherapy [19] or bevacizumab use [20], and baseline neutrophil-to-lymphocyte (NLR) ratio [21] and platelet-tolymphocyte ratio [22]. The second group of predictors belongs to "evolving or developing" determinants that arise concurrently with immunotherapy treatment, which may be subject to exploitation by external factors. These predictive markers include but are not limited to change in gut microbiome [23], change in NLR [24] and lymphocyte-tomonocyte ratio [25], and immune-related adverse events (irAEs) $[26,27]$. Based on current data, no new biomarkers have been identified in long-term survivors specifically. In the aforementioned nivolumab phase I CA209-003 trial [6], the majority of the long-term survivors were current smokers (14 out of 16 patients). In subgroup analysis, the 5-year OS rate was higher in patients with PDL-1 expression $>50 \%$ ( $43 \%$ vs. $20 \%$ in PDL- $1<1$, and $23 \%$ in PDL- $1 \geq 1 \%$ ). Most 


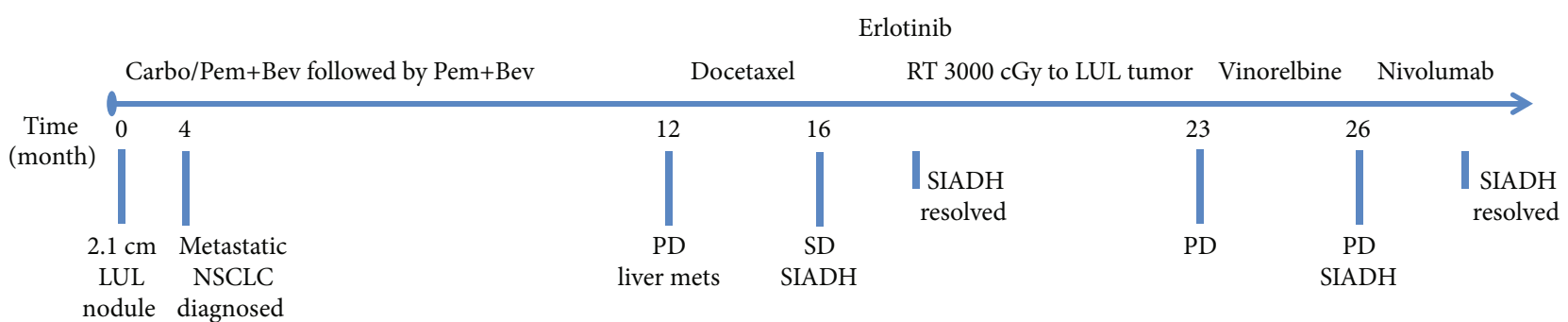

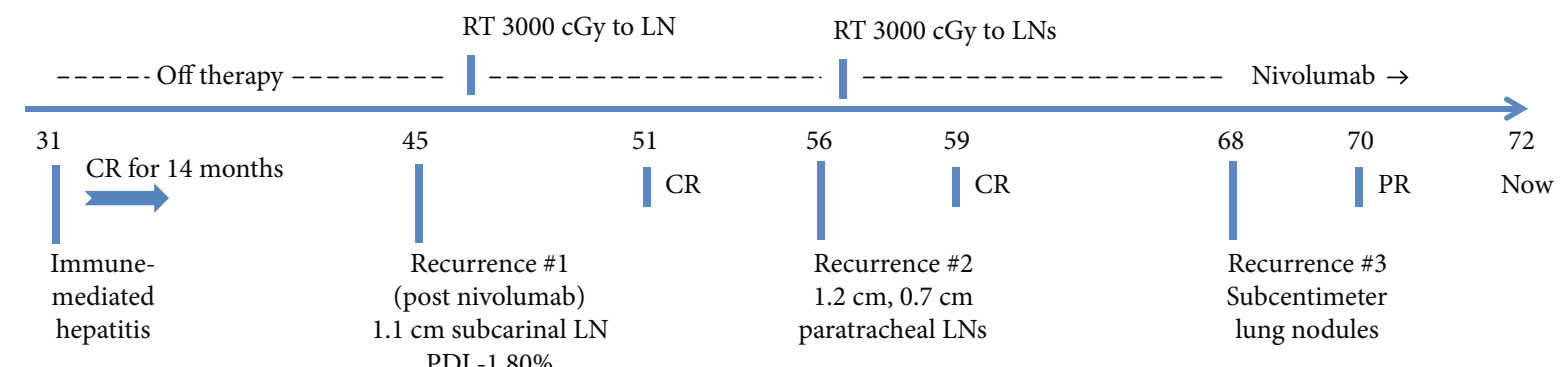

FIGURE 1: Treatment timeline. ${ }^{*}$ The numbers below the axis represent the number of months since the initial presentation. Abbreviations: NSCLC_non-small-cell lung cancer; Carbo-carboplatin; Pem-pemetrexed; Bev-bevacizumab; LUL-left upper lobe of the lung; $\mathrm{PD}$ - progression of disease; SD—stable disease; CR—complete response; PR-partial response; LN-lymph node; PDL-1-programmed death ligand-1; SIADH_-syndrome of inappropriate antidiuretic hormone secretion.

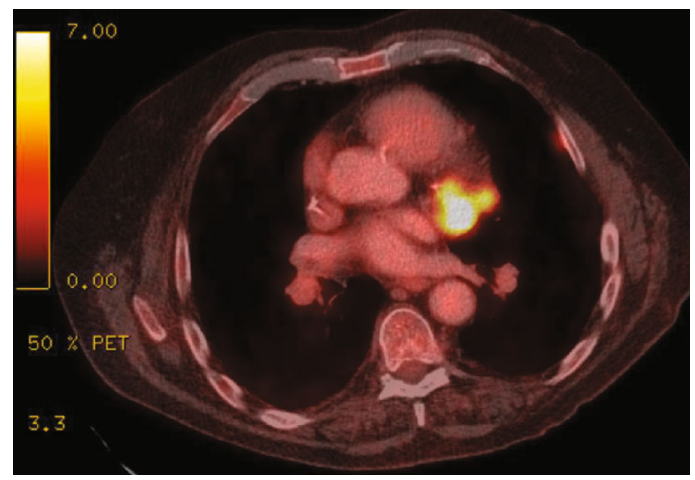

(a)

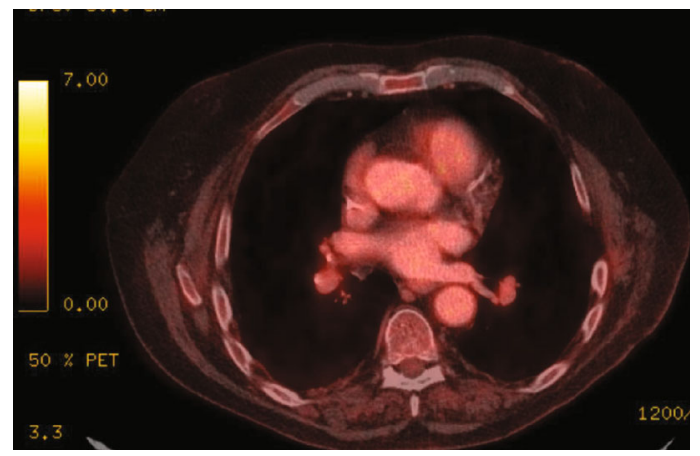

(c)

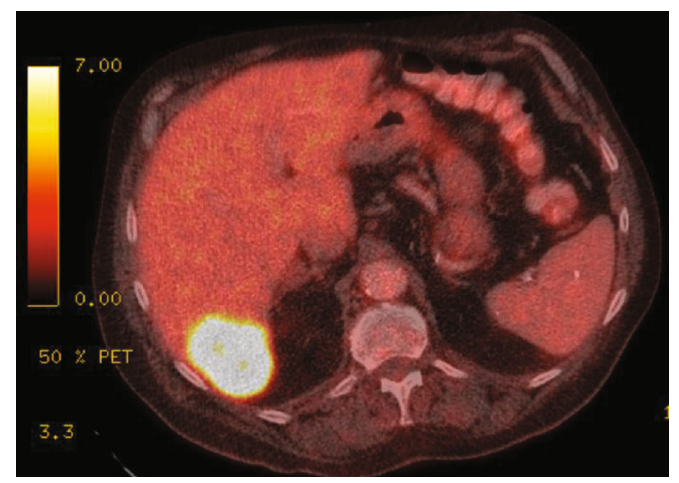

(b)

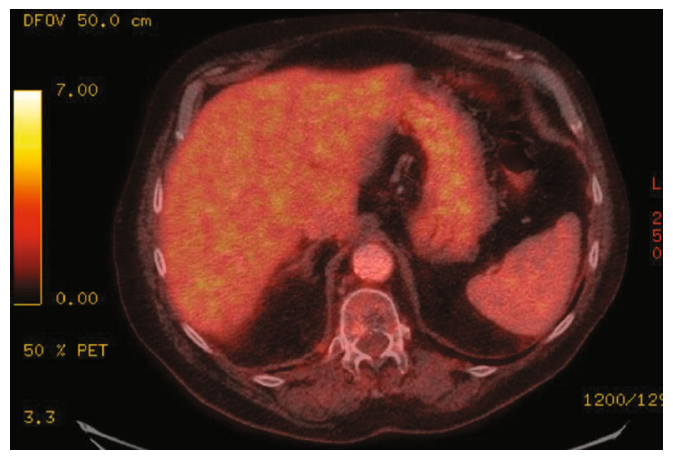

(d)

FIGURE 2: PET/CT images showing increased metabolic activity in the mediastinum (a) and in the liver (b) prior to initiating nivolumab. Resolution of FDG-avid lesions in the mediastinum (c) and in the liver (d) 10 months after discontinuation of nivolumab. 
TABLE 1: Long-term outcomes of patients treated with immune checkpoint inhibitors in metastatic NSCLC.

\begin{tabular}{|c|c|c|}
\hline & Characteristics & Outcomes and exceptional responders \\
\hline \multicolumn{3}{|c|}{ Clinical trials (5 yr data) } \\
\hline Phase 1 (CA 209-003) & & \\
\hline & & $\begin{array}{c}\text { Median OS } 9.9 \mathrm{~m} \\
5 \text {-year OS } 16 \%(43 \% \text { for PDL-1 } \geq 50 \%)\end{array}$ \\
\hline Nivolumab & 129 patients & For 5-year survivors: \\
\hline 2nd line; Ref. [6] & Median follow-up: $58 \mathrm{~m}$ & $\begin{array}{l}14 / 16 \text { current or former smokers, } 10 / 16 \text { had received prior RT, } \\
12 / 16 \text { no further tx after stopping Nivo and without PD } \\
\text { PDL- } 1 \geq 1 \% \text { in } 7, \geq 50 \% \text { in } 5,<1 \% \text { in } 3 \text { pts }\end{array}$ \\
\hline \multicolumn{3}{|l|}{ Phase Ib (Keynote 001) } \\
\hline $\begin{array}{l}\text { Pembrolizumab } \\
\text { 1st line or later; Ref. [8] }\end{array}$ & $\begin{array}{l}101 \text { tx naïve } \\
449 \text { previously treated } \\
\text { Median follow-up: } 60.6 \mathrm{~m}\end{array}$ & $\begin{array}{c}\text { Treatment naïve: } \\
\text { Median DOR } 16.8 \mathrm{~m} \text {; CR 3\% (3 patients) } \\
\text { Median OS } 22.3 \mathrm{~m} \\
\text { 5-year OS 23.2\% (29.6\% for PDL-1 } \geq 50 \%) \\
\text { Previously treated: } \\
\text { Median DOR 38.9 m; CR 1.1\% (5 patients) } \\
\text { Median OS } 10.5 \mathrm{~m} \text { (15.5 m for PDL-1 } \geq 50 \%) \\
\text { 5-year OS } 15.6 \% \text { (25\% for PDL-1 } \geq 50 \%)\end{array}$ \\
\hline
\end{tabular}

Clinical trials (3yr data)

Phase III (CheckMate 017, 057)

Nivolumab vs. Doc

2nd line; Ref. [35]

Phase III (Keynote 010)

Pembrolizumab vs. Doc

2nd line or later; Ref. [49]

Phase II (POPLAR)

Atezolizumab vs. Doc

2nd line; Ref. [50]
854 patients

Median follow-up: $40.3 \mathrm{~m}$

1033 patients

Median follow-up: $42.6 \mathrm{~m}$

Median DOR $23.8 \mathrm{~m}$

CR $6 \%$ for those alive at 3 years

3-year OS: $17 \%$ (vs. $8 \%$ Doc), $8 \%$ (vs. $2 \%$ ) for patients with liver metastases

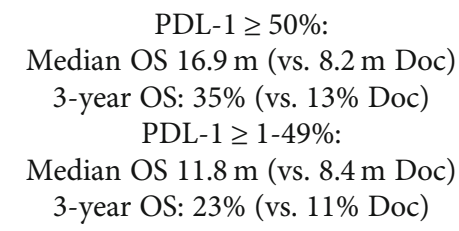

Median DOR: $22.3 \mathrm{~m}$ (vs. $7.2 \mathrm{~m}$ docetaxel) 3-year OS:

ITT $16.6 \%$ (vs. $10 \%$ docetaxel)

IHC TC3 or IC3 $37.5 \%$ (vs. $14.9 \%$ )

IHC TC $2 / 3$ or IC $2 / 321.2 \%$ (vs. $9.9 \%$ )

Minimum 3-year follow-up
Nivolumab-induced pneumonitis after 3 doses; following discontinuation of the drug, the patient continued to have complete remission for 14 months

Radiation-induced pneumonitis

CR after 6 cycles, ongoing after 2 years of continuous nivolumab

PR after 6 cycles; CR after 13 cycles (10 weeks after RT)

Nivo discontinued after cycle 17 due to pancreatitis; still in CR almost 2 years since discontinuation 2nd line; Ref. [31]
47 male; adenocarcinoma Current smoker

SBRT to LNs while on Nivo

\section{Case reports}

3rd line; Ref. [28]

Nivolumab

2nd line; Ref. [29]

80 male; squamous cell ca

T (3 months prior to Nivo)

$$
\text { PDL-1 positive }>5 \%
$$

o

Abbreviations: OS-overall survival; $\mathrm{m}$-months; tx-treatment; DOR-duration of response; CR-complete response; PD—progressive disease; ITT_intention to treat; Nivo-nivolumab; doc-docetaxel; LN-lymph node; ca-carcinoma; NSCLC_non-small-cell lung cancer; RT-radiotherapy; SBRT—stereotactic body radiation therapy; IHC_immunohistochemistry; TC—-tumor cells; IC-tumor-infiltrating immune cells; Mut/Mb-mutations per megabase; PDL-1-programmed death ligand-1.

interestingly, $75 \%$ of the long-term survivors received nivolumab for no more than two years, required no further cancer therapy afterwards, and remained without disease progression as of their last follow-up visit (median follow-up of 58 months). Majority of this cohort (75\%) had partial response as their best response, and none had a CR.

Case reports of exceptional responders to ICI in NSCLC are rare and possibly underreported (Table 1) [28-33]. Our 
case is unique in several aspects. First, it is a real-world scenario of an exceptional responder to immunotherapy outside of a clinical trial. Second, our patient has been diagnosed with metastatic lung cancer more than 5.5 years and continues to do well with very limited disease burden following eight lines of therapy, including RT. Third, he exhibited many of the predictive markers mentioned above, including high PDL-1 expression (80\%), smoking history, prior RT and bevacizumab use, and development of immune-mediated hepatitis requiring nivolumab to be held. Although he continues to smoke daily, it currently remains unclear if active smoking has any role in potentiating response to ICI. Interestingly, unlike what is reported in the literature on poor responses to ICI in liver metastasis [34], our patient had a CR to nivolumab and his disease never recurred in his liver despite having a relatively large liver metastasis (Figure 2), possibly due to the activation of the immune system that resulted in hepatitis. Notably, in phase III CheckMate 017 and 057 trials, nivolumab resulted in improved OS compared with docetaxel in patients with liver metastases, and their immunemediated hepatic adverse events were higher than the overall pooled population (10\% vs. 6\%) [35]. Fourth, the clinical course following nivolumab discontinuation is interesting as he achieved CR lasting 14 months after a short course of nivolumab. Notably, the prolonged course of steroid taper for his hepatitis did not affect his duration of response nor did it affect his second response to nivolumab upon rechallenge. Ongoing responses following discontinuation of immunotherapy is a well-known phenomenon and has been documented in clinical trials, including the nivolumab trial above $[6,36]$. Fifth, he was noted to have a RET rearrangement on repeat biopsy. In contrast to our case, a recent case series by Offin et al. [37] showed that majority of RETrearranged lung cancers have low PDL-1 expression and low TMB, and exhibit poor responses to ICIs. However, only one patient in that series had PDL-1 expression $>50 \%$, and hence no conclusions as to how to sequence targeted therapies in such patients can be drawn. In an ongoing global registry (IMMUNOTARGET), it was noted that ICI efficacy in oncogenic-driven lung cancers is inconsistent and depends on smoking history, PDL-1 expression, and the type of mutation (less benefit in RET/EGFR/ALK patients) [38]. In our case, we did not use a RET inhibitor as the patient's cancer recurrences were mostly localized, and it was thought that radiotherapy would provide the best local control with potential for greater disease-free and treatment-free intervals. Upon his last relapse, immunotherapy rechallenge was also more appealing given the patient's initial complete durable response and high PDL-1 expression. As expected, his local disease recurrences were successfully treated with radiation therapy, with responses lasting 9 and 12 months after each RT. Finally, rechallenge with nivolumab following his third tumor recurrence resulted in rapid partial response after four cycles. In the future, we plan to use a RET inhibitor as the next line of therapy once he progresses on nivolumab.

Optimal duration of immunotherapy in responders remains unclear and is an area of active investigation. A randomized trial of continuous (76 patients) vs. 1-year fixed duration (87 patients) nivolumab in advanced NSCLC (CheckMate 153, $n=220$ ) showed significant improvement in progression-free survival (hazard ratio (HR) $0.42,95 \%$ confidence interval (CI): $0.25,0.71)$ and a trend towards better OS (HR 0.63, 95\% CI: 0.33, 1.20) favoring the continuous nivolumab arm [39]. However, it should be noted that the continuous arm had a higher percentage of PDL-1 expression $>50 \%(28 \%$ vs. $23 \%)$ and higher rates of $\mathrm{CR}$ ( $10 \%$ vs. $2 \%)$ at baseline (1-year randomization mark) compared to the 1-year fixed duration arm. Notably, two patients who achieved CR in the 1-year treatment arm recurred 6 and 13 months after stopping treatment. Retreatment with nivolumab after disease progression while off therapy resulted in response in the majority of patients, although the duration of response to the rechallenge was short for those with early progression ( $<6$ months) [39].

Immune-related adverse events are major causes of ICI interruption or discontinuation, complicating the subsequent management of patients, particularly those who have achieved durable clinical benefit. irAEs have been generally associated with better ICI efficacy as well as increased overall survival [26, 27, 40]. Although the optimal oncologic management of patients who discontinue therapy due to irAEs is unknown, current data $[6,28]$, including our case report, suggest that patients who achieve a $\mathrm{CR}$ are more likely to have prolonged duration of response off therapy. Whether resuming immunotherapy (if prior irAE grade $\leq 2$ ) or adding chemotherapy in the absence of measurable metastatic disease further extends duration of response or even achieves the ultimate goal of cure is currently unknown. Limited data at this time suggest that it may be reasonable to rechallenge patients with immunotherapy upon recurrence $[6,33,41-44]$, or treat oligometastatic recurrences with surgery or radiation therapy, with lower threshold to initiate systemic therapy following multiple recurrences or in those with higher burden of disease [6]. Furthermore, increased use of liquid biopsies (such as circulating tumor DNA) [45, 46], tumor markers (such as CEA, CA125) [47, 48], and blood parameters (such as NLR) [46] as biomarkers of response or relapse in ICItreated patients will further identify early responders, discern CRs from occult disease, and guide therapy in patients with early progression.

\section{Conclusion}

Exceptional responders to immune checkpoint inhibitors are uncommon in metastatic NSCLC. Additionally, the optimal duration of immunotherapy in patients who achieve complete remission remains unknown. Our case report adds to the limited data in the management of exceptional responders following discontinuation of immunotherapy. Treatment of oligometastatic recurrences with localized therapy and rechallenge with immunotherapy may increase disease control and extend overall survival. Further understanding of tumor microenvironment and tumor/immune system interactions may help identify better predictors of response and further improve patients' outcomes. 


\section{Conflicts of Interest}

The authors declare that they have no conflicts of interest.

\section{References}

[1] D. B. Doroshow, M. F. Sanmamed, K. Hastings et al., "Immunotherapy in non-small cell lung cancer: facts and hopes," Clinical Cancer Research, vol. 25, no. 15, pp. 4592-4602, 2019.

[2] J. Remon, M. J. Ahn, N. Girard et al., "Advanced-stage nonsmall cell lung cancer: advances in thoracic oncology 2018," Journal of Thoracic Oncology, vol. 14, no. 7, pp. 1134-1155, 2019.

[3] J. Li, Q. He, X. Yu, K. Khan, X. Weng, and M. Guan, "Complete response associated with immune checkpoint inhibitors in advanced non-small-cell lung cancer: a meta-analysis of nine randomized controlled trials," Cancer Management and Research, vol. 11, pp. 1623-1629, 2019.

[4] R. Chen, X. Hou, L. Yang, and D. Zhao, "Comparative efficacy and safety of first-line treatments for advanced non-small cell lung cancer with immune checkpoint inhibitors: a systematic review and meta-analysis," Thoracic Cancer, vol. 10, no. 4, pp. 607-623, 2019.

[5] A. Anichini, E. Tassi, G. Grazia, and R. Mortarini, "The nonsmall cell lung cancer immune landscape: emerging complexity, prognostic relevance and prospective significance in the context of immunotherapy," Cancer Immunology, Immunotherapy, vol. 67, no. 6, pp. 1011-1022, 2018.

[6] S. Gettinger, L. Horn, D. Jackman et al., "Five-year follow-up of nivolumab in previously treated advanced non-small-cell lung cancer: results from the CA209-003 study," Journal of Clinical Oncology, vol. 36, no. 17, pp. 1675-1684, 2018.

[7] K. Cetin, D. S. Ettinger, Y. J. Hei, and C. D. O'Malley, "Survival by histologic subtype in stage IV nonsmall cell lung cancer based on data from the surveillance, epidemiology and end results program," Clinical Epidemiology, vol. 3, pp. 139-148, 2011.

[8] E. B. Garon, M. D. Hellmann, N. A. Rizvi et al., "Five-year overall survival for patients with advanced non-small-cell lung cancer treated with pembrolizumab: results from the phase I KEYNOTE-001 study," Journal of Clinical Oncology, vol. 37, no. 28, pp. 2518-2527, 2019.

[9] E. Nadal, B. Massuti, M. Domine, R. Garcia-Campelo, M. Cobo, and E. Felip, "Immunotherapy with checkpoint inhibitors in non-small cell lung cancer: insights from longterm survivors," Cancer Immunology, Immunotherapy, vol. 68, no. 3, pp. 341-352, 2019.

[10] H. El-Osta and S. Jafri, "Predictors for clinical benefit of immune checkpoint inhibitors in advanced non-small-cell lung cancer: a meta-analysis," Immunotherapy, vol. 11, no. 3, pp. 189-199, 2019.

[11] R. S. Herbst, J. C. Soria, M. Kowanetz et al., "Predictive correlates of response to the anti-PD-L1 antibody MPDL3280A in cancer patients," Nature, vol. 515, no. 7528, pp. 563-567, 2014.

[12] J. Kim, J. Cho, M. H. Lee, and J. H. Lim, "Relative Efficacy of Checkpoint Inhibitors for Advanced NSCLC According to Programmed Death-Ligand-1 Expression: A Systematic Review and Network Meta- Analysis," Scientific Reports, vol. 8, no. 1, article 11738, 2018.

[13] E. B. Garon, N. A. Rizvi, R. Hui et al., "Pembrolizumab for the treatment of non-small-cell lung cancer," The New England Journal of Medicine, vol. 372, no. 21, pp. 2018-2028, 2015.
[14] H. Borghaei, L. Paz-Ares, L. Horn et al., "Nivolumab versus docetaxel in advanced nonsquamous non-small-cell lung cancer," The New England Journal of Medicine, vol. 373, no. 17, pp. 1627-1639, 2015.

[15] N. A. Rizvi, M. D. Hellmann, A. Snyder et al., "Cancer immunology. Mutational landscape determines sensitivity to PD-1 blockade in non-small cell lung cancer," Science, vol. 348, no. 6230, pp. 124-128, 2015.

[16] D. T. Le, J. N. Uram, H. Wang et al., "PD-1 blockade in tumors with mismatch-repair deficiency," The New England Journal of Medicine, vol. 372, no. 26, pp. 2509-2520, 2015.

[17] P. C. Tumeh, C. L. Harview, J. H. Yearley et al., "PD-1 blockade induces responses by inhibiting adaptive immune resistance," Nature, vol. 515, no. 7528, pp. 568-571, 2014.

[18] Q. Huang, H. Zhang, J. Hai et al., "Impact of PD-L1 expression, driver mutations and clinical characteristics on survival after anti-PD-1/PD-L1 immunotherapy versus chemotherapy in non-small-cell lung cancer: a meta-analysis of randomized trials," Oncoimmunology, vol. 7, no. 12, article e1396403, 2018.

[19] N. Shaverdian, A. E. Lisberg, K. Bornazyan et al., "Previous radiotherapy and the clinical activity and toxicity of pembrolizumab in the treatment of non-small-cell lung cancer: a secondary analysis of the KEYNOTE-001 phase 1 trial," The Lancet Oncology, vol. 18, no. 7, pp. 895-903, 2017.

[20] R. Giannicola, G. D'arrigo, P. Pastina et al., "Correlation of survival of NSCLC patients given nivolumab with treatment related-eosinophilia, autoimmunity and previous bevacizumab containing regimens," Journal of Clinical Oncology, vol. 36, article e21008, Supplement 15, 2018.

[21] S. J. Bagley, S. Kothari, C. Aggarwal et al., "Pretreatment neutrophil-to-lymphocyte ratio as a marker of outcomes in nivolumab-treated patients with advanced non-small-cell lung cancer," Lung Cancer, vol. 106, pp. 1-7, 2017.

[22] S. Diem, S. Schmid, M. Krapf et al., "Neutrophil-to-lymphocyte ratio (NLR) and platelet-to-lymphocyte ratio (PLR) as prognostic markers in patients with non-small cell lung cancer (NSCLC) treated with nivolumab," Lung Cancer, vol. 111, pp. 176-181, 2017.

[23] B. Routy, E. Le Chatelier, L. Derosa et al., "Gut microbiome influences efficacy of PD-1-based immunotherapy against epithelial tumors," Science, vol. 359, no. 6371, pp. 91-97, 2018.

[24] T. Kiriu, M. Yamamoto, T. Nagano et al., "The time-series behavior of neutrophil-to-lymphocyte ratio is useful as a predictive marker in non-small cell lung cancer," PLoS One, vol. 13, no. 2, article e0193018, 2018.

[25] K. Sekine, S. Kanda, Y. Goto et al., "Change in the lymphocyteto-monocyte ratio is an early surrogate marker of the efficacy of nivolumab monotherapy in advanced non-small-cell lung cancer," Lung Cancer, vol. 124, pp. 179-188, 2018.

[26] A. Cortellini, R. Chiari, B. Ricciuti et al., "Correlations between the immune-related adverse events spectrum and efficacy of anti-PD1 immunotherapy in NSCLC patients," Clinical Lung Cancer, vol. 20, no. 4, pp. 237-247.e1, 2019.

[27] K. Haratani, H. Hayashi, Y. Chiba et al., "Association of immune-related adverse events with nivolumab efficacy in non-small-cell lung cancer," JAMA Oncology, vol. 4, no. 3, pp. 374-378, 2018.

[28] H. Li, W. Ma, K. Y. Yoneda et al., "Severe nivolumab-induced pneumonitis preceding durable clinical remission in a patient with refractory, metastatic lung squamous cell cancer: a case 
report," Journal of Hematology \& Oncology, vol. 10, no. 1, p. 64, 2017.

[29] G. Lazzari, A. Terlizzi, G. Porrazzo et al., "Effective nivolumab sequential thoracic radiotherapy in elderly patients with advanced squamous cell lung cancer: did radiation therapy play a role? A case report," OncoTargets and Therapy, vol. 11, pp. 4621-4629, 2018.

[30] D. Keohane and G. P. Fitzgerald, "The changing face of cancer treatments," BMJ Case Reports, 2018, bcr-2018-224784.

[31] C. Britschgi, O. Riesterer, I. A. Burger, M. Guckenberger, and A. Curioni-Fontecedro, "Report of an abscopal effect induced by stereotactic body radiotherapy and nivolumab in a patient with metastatic non-small cell lung cancer," Radiation Oncology, vol. 13, no. 1, p. 102, 2018.

[32] B. McCullar, T. Alloway, and M. Martin, "Durable complete response to nivolumab in a patient with HIV and metastatic non-small cell lung cancer," Journal of Thoracic Disease, vol. 9, no. 6, pp. E540-E542, 2017.

[33] R. Tay, A. Prelaj, and R. Califano, "Immune checkpoint blockade for advanced non-small cell lung cancer: challenging clinical scenarios," Journal of Thoracic Disease, vol. 10, Supplement 13, pp. S1494-S1502, 2018.

[34] P. C. Tumeh, M. D. Hellmann, O. Hamid et al., "Liver metastasis and treatment outcome with anti-PD-1 monoclonal antibody in patients with melanoma and NSCLC," Cancer Immunology Research, vol. 5, no. 5, pp. 417-424, 2017.

[35] E. E. Vokes, N. Ready, E. Felip et al., "Nivolumab versus docetaxel in previously treated advanced non-small-cell lung cancer (CheckMate 017 and CheckMate 057): 3-year update and outcomes in patients with liver metastases," Annals of Oncology, vol. 29, no. 4, pp. 959-965, 2018.

[36] L. Horn, D. R. Spigel, E. E. Vokes et al., "Nivolumab versus docetaxel in previously treated patients with advanced nonsmall-cell lung cancer: two-year outcomes from two randomized, open-label, phase III trials (CheckMate 017 and CheckMate 057)," Journal of Clinical Oncology, vol. 35, no. 35, pp. 3924-3933, 2017.

[37] M. Offin, R. Guo, S. L. Wu et al., "Immunophenotype and response to immunotherapy ofRET-Rearranged lung cancers," JCO Precision Oncology, vol. 3, no. 3, pp. 1-8, 2019.

[38] J. Mazieres, A. E. Drilon, L. Mhanna et al., "Efficacy of immune-checkpoint inhibitors (ICI) in non-small cell lung cancer (NSCLC) patients harboring activating molecular alterations (ImmunoTarget)," Journal of Clinical Oncology, vol. 36, Supplement 15, p. 9010, 2018.

[39] D. R. Spigel, M. Mcleod, M. A. Hussein et al., “1297ORandomized results of fixed-duration (1-yr) vs continuous nivolumab in patients (pts) with advanced non-small cell lung cancer (NSCLC)," Annals of Oncology, vol. 28, Supplement 5, 2017.

[40] A. Qin, L. Street, K. Cease et al., "Clinical determinants of durable clinical benefit of pembrolizumab in veterans with advanced non-small-cell lung cancer," Clinical Lung Cancer, vol. 18, no. 5, pp. 559-564, 2017.

[41] A. Bernard-Tessier, C. Baldini, P. Martin et al., "Outcomes of long-term responders to anti-programmed death 1 and antiprogrammed death ligand 1 when being rechallenged with the same anti-programmed death 1 and anti-programmed death ligand 1 at progression," European Journal of Cancer, vol. 101, pp. 160-164, 2018.
[42] L. Horn, S. N. Gettinger, M. S. Gordon et al., "Safety and clinical activity of atezolizumab monotherapy in metastatic non- small-cell lung cancer: final results from a phase I study," European Journal of Cancer, vol. 101, pp. 201-209, 2018.

[43] K. Fujita, N. Uchida, O. Kanai, M. Okamura, K. Nakatani, and T. Mio, "Retreatment with pembrolizumab in advanced nonsmall cell lung cancer patients previously treated with nivolumab: emerging reports of 12 cases," Cancer Chemotherapy and Pharmacology, vol. 81, no. 6, pp. 1105-1109, 2018.

[44] K. Fujita, N. Uchida, Y. Yamamoto et al., "Retreatment with anti-PD-L1 antibody in advanced non-small cell lung cancer previously treated with anti-PD-1 antibodies," Anticancer Research, vol. 39, no. 7, pp. 3917-3921, 2019.

[45] P. Hofman, S. Heeke, C. Alix-Panabieres, and K. Pantel, "Liquid biopsy in the era of immuno-oncology: Is it ready for prime-time use for cancer patients?," Annals of Oncology, vol. 30, no. 9, pp. 1448-1459, 2019.

[46] F. Passiglia, A. Galvano, M. Castiglia et al., "Monitoring blood biomarkers to predict nivolumab effectiveness in NSCLC patients," Therapeutic Advances in Medical Oncology, vol. 11, 2019.

[47] M. G. Dal Bello, R. A. Filiberti, A. Alama et al., "The role of CEA, CYFRA21-1 and NSE in monitoring tumor response to Nivolumab in advanced non-small cell lung cancer (NSCLC) patients," Journal of Translational Medicine, vol. 17, no. 1, p. 74, 2019.

[48] M. Zhuo, H. Chen, T. Zhang et al., "The potential predictive value of circulating immune cell ratio and tumor marker in atezolizumab treated advanced non-small cell lung cancer patients," Cancer Biomarkers, vol. 22, no. 3, pp. 467-476, 2018.

[49] R. S. Herbst, E. B. Garon, D. W. Kim et al., "Long-term survival in patients (pts) with advanced NSCLC in the KEYNOTE-010 study overall and in pts who completed two years of pembrolizumab (pembro)," in European Society for Medical Oncology, Munich, Germany, October 2018.

[50] J. Mazières, K. Park, C. Lewanski et al., “136PD_PR 3-year survival and duration of response in randomized phase II study of atezolizumab (atezo) vs docetaxel (doc) in 2L+ NSCLC (POPLAR)," Journal of Thoracic Oncology, vol. 13, no. 4, p. S79, 2018. 


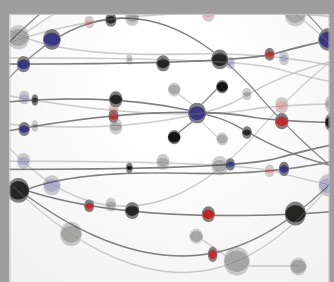

The Scientific World Journal
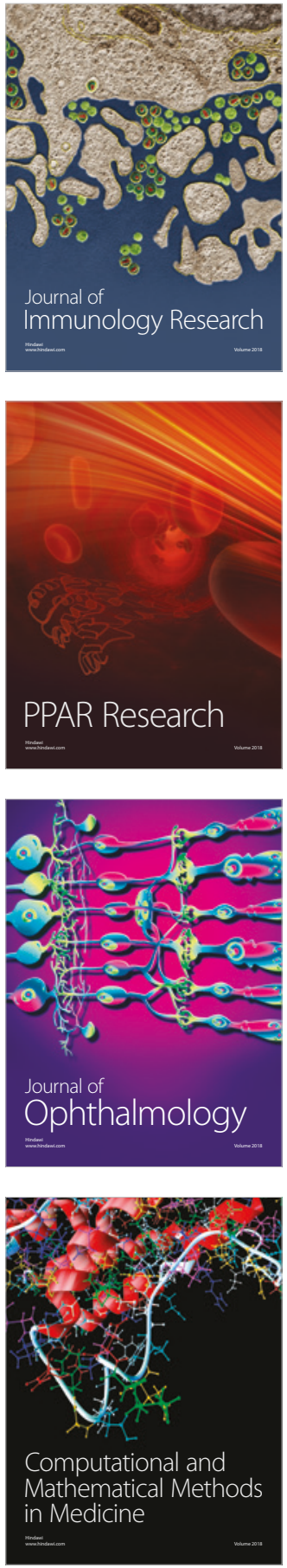

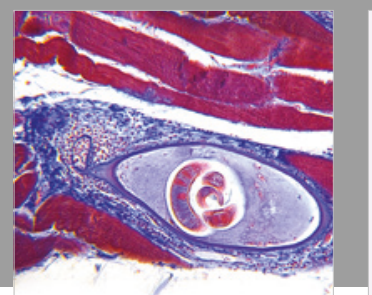

Gastroenterology Research and Practice

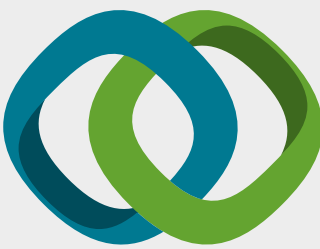

\section{Hindawi}

Submit your manuscripts at

www.hindawi.com
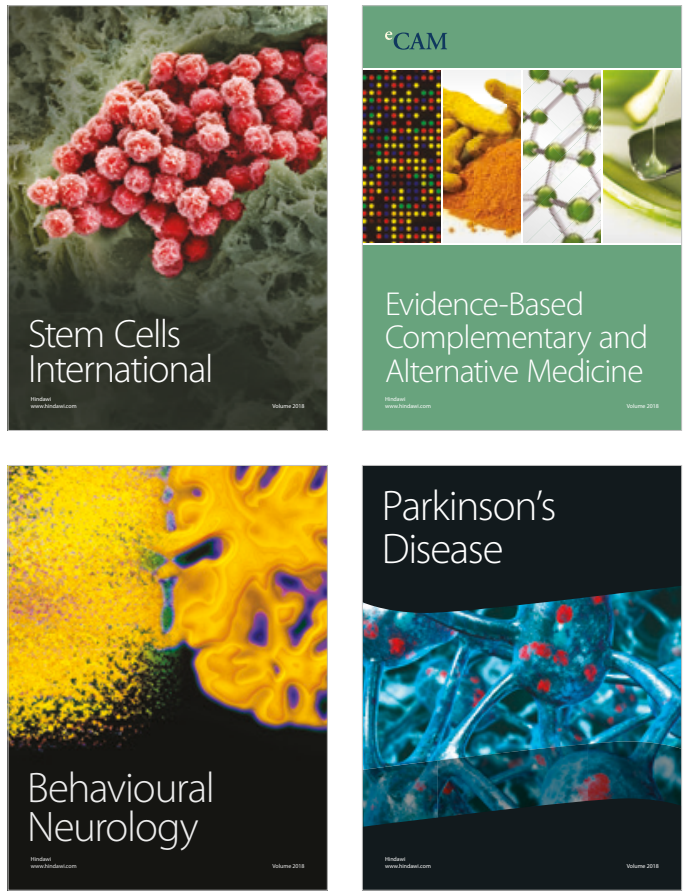

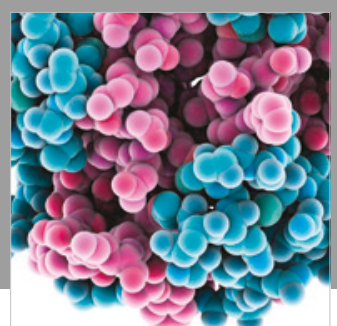

ournal of

Diabetes Research

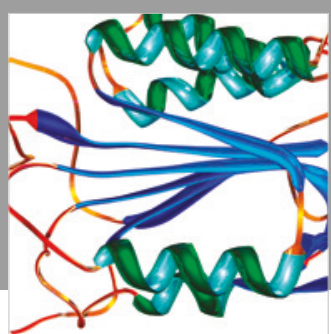

Disease Markers
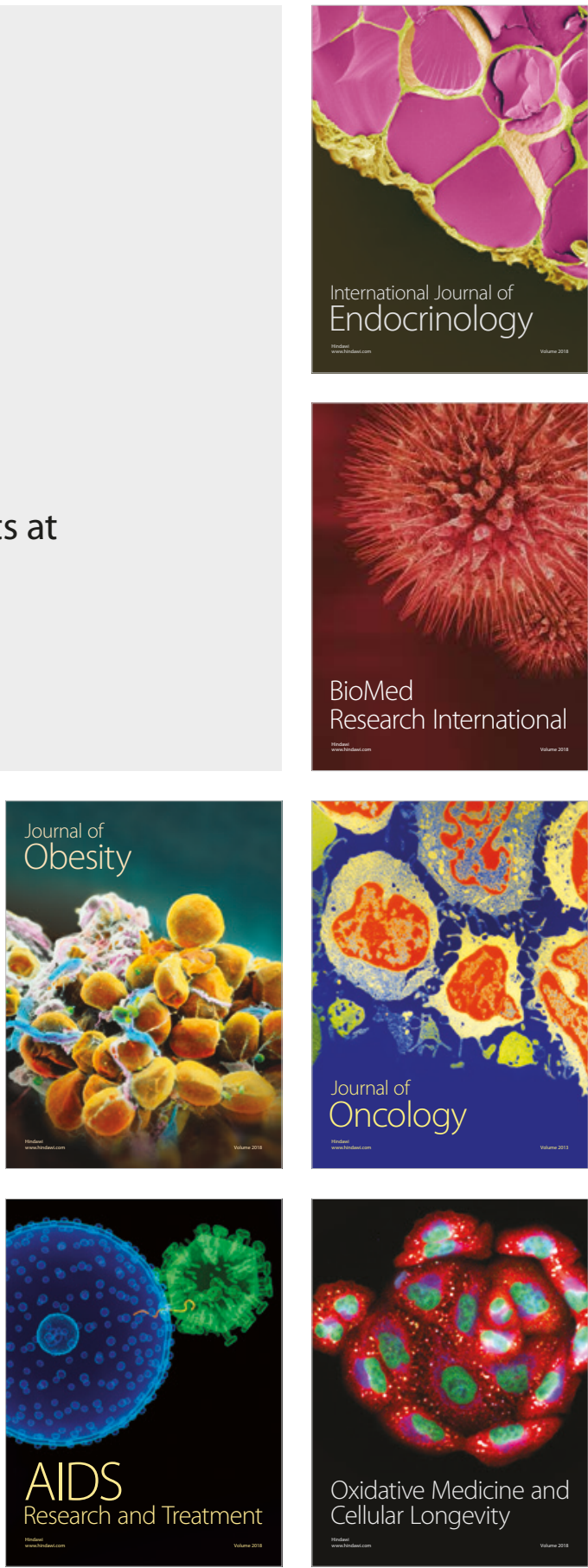